\title{
Norois
}

Environnement, aménagement, société

$217 \mid 2010 / 4$

Interroger les processus de valorisation des espaces urbains

\section{Nouveaux lieux du commerce et transformation des pratiques de consommation dans les villes algériennes. Étude de cas à El Eulma et Onama/El- Ghazi (Constantine)}

New market areas and transformations of consumering practices in Algerian cities. Case study in El Eulma and Onama/El-Ghazi (Constantina)

Pierre Bergel et Nadia Kerdoud

\section{OpenEdition}

\section{Édition électronique}

URL : http://journals.openedition.org/norois/3504

DOI : 10.4000/norois.3504

ISBN : 978-2-7535-1565-9

ISSN : $1760-8546$

Éditeur

Presses universitaires de Rennes

Édition imprimée

Date de publication : 15 décembre 2010

Pagination : 75-89

ISBN : 978-2-7535-1340-2

ISSN : 0029-182X

\section{Référence électronique}

Pierre Bergel et Nadia Kerdoud, « Nouveaux lieux du commerce et transformation des pratiques de consommation dans les villes algériennes. Étude de cas à El Eulma et Onama/El-Ghazi

(Constantine) », Norois [En ligne], 217 | 2010/4, mis en ligne le 31 décembre 2012, consulté le 03 mai 2019. URL : http://journals.openedition.org/norois/3504 ; DOI : 10.4000/norois.3504 


\title{
Nouveaux LIEUX DU COMMERCE ET TRANSFORMATION DES PRATIQUES DE CONSOMMATION DANS LES VILLES ALGÉRIENNES Étude de cas à El Eulma et Onama/El-Ghazi (Constantine)
}

\author{
Pierre Bergel \\ ESO-Caen UMR ESO 6590 \\ (Université de Caen Basse-Normandie),
}

Maison de la recherche en sciences humaines, Esplanade de la Paix - 14032 CAEN Cedex, France pierre.bergel@unicaen.fr

NADia KeRdoud

ESO-Caen UMR ESO 6590

(Université Mentouri),

Faculté des Sciences de la terre et de l'aménagement du territoire, BP 325 Route Ain El Bey - Constantine 25017, Algérie

nadia.kerdoud@gmail.com

\section{RÉSUMÉ}

Les années 2000 ont vu de nouveaux lieux du commerce se développer à la périphérie des villes algériennes. Si l'Algérie suit une tendance observable sur l'ensemble du continent africain ou au Moyen-Orient, les transformations y semblent plus rapides dans la mesure où le pays a été isolé durant une décennie pour cause de guerre intérieure. Dans ces lieux qui empruntent à la fois au bazar traditionnel, au grand magasin à l'européenne et au mall nord-américain, la clientèle trouve des biens d'importation variés à des prix suffisamment abordables pour satisfaire son appétit de consommation.

À partir de deux terrains d'enquête urbains situés dans le Nord-Est algérien, Constantine et El Eulma, le présent article se propose de poursuivre les recherches sur la "mondialisation par le bas » qui resserre les liens entre économie de bazar et commerce planétaire. Du côté de la clientèle, sont décrites des pratiques de "shopping " jusqu'ici peu répandues en Algérie. Dans ces nouveaux espaces de la consommation mondialisée, sont testés de nouveaux rapports à soi, aux autres et à la marchandise, à leur tour alimentés par les mutations de la famille et de la société algérienne ainsi que par une transformation du rôle des femmes.

MoTS CLÉ : nowveaux lieux du commerce - changement social - mondialisation - pratiques de consommation - Algérie - Constantine - El Eulma

\footnotetext{
ABSTRACT

New market areas and transformations of consumering practices in Algerian cities. Case study in El Eulma and Onama/El-Ghazi (Constantina)

Since the beginning of the 2000's, new market areas are quickly growing up at the boundaries of several algerian cities. Although Algeria follows a general trend common in the rest of Maghreb and Middle-East, changes are more violent in counterpart of the 1990's civil war which insulated society and economy for ten years. Those new market areas partly
} 
inspired from european general stores, north-american shopping malls and traditional oriental bazaar offer to customers needs of mass consumption with various foreign wares.

Based on investigations carried out in two north-eastern algerian cities, Castantina and El-Eulma, the aim of this paper is to carry on a research about lower and ordinary globalization which narrow the gap between traditional souk bazaar and global trade. For their part, shoppers build new relationships to themselves, other customers or to the wares with new consumering practices and window shopping unseen in Algeria before now. Quick transformations in algerian families and society build up those new consumering practices and improve women's position.

KEY WORDS : new market areas - globalization - social change - globalization - consumering practices - Algeria - Constantina - El Eulma

L'activité commerciale est un déterminant de premier ordre dans les pratiques et dans les appropriations spatiales des citadins. De ce fait, le commerce urbain ne remplit pas seulement une fonction économique. Autour de l'échange marchand se nouent des chapelets d'interactions où les frontières entre licite et interdit, bienséant et scandaleux varient selon les circonstances, les catégories sociales, les générations. Les lieux du commerce constituent donc une scène sociale (Hancock, 2008) où le chaland, le passant et le commerçant sont à la fois producteurs et spectateurs d'un espace en permanente reconfiguration.

Dans ce cadre, les villes algériennes présentent trois particularités intéressantes. Méditerranéennes, elles accordent une place prépondérante à l'activité commerciale (Troin, 1985; Côte, 2005). L'économie de bazar et de marché font converger la clientèle en certains lieux (souks, grandes places) et selon certaines temporalités (fin de semaine, fêtes religieuses, rentrée des classes). Cette économie commerçante produit de la richesse et de l'emploi et peut en outre susciter des aménagements. Palliant les carences de la puissance publique, propriétaires ou gérants de boutiques construisent ou modifient des immeubles, aménagent des passages couverts, etc. Cette valorisation est matérielle mais elle présente également une portée symbolique, l'embellissement des lieux visant à convaincre la clientèle qu'elle renforcera son statut et sa position à fréquenter telle boutique ou tel quartier.

En Algérie, ces lieux sont également intéressants du point de vue de leur histoire récente, particulièrement heurtée. Succédant à une fermeture relative (années 1960-1970), puis à la « décennie noire » de la guerre civile (1990), lieux et pratiques du commerce urbain algérien se reconfigurent depuis les années 2000. Comme dans d'autres pays du Sud, mais avec peut-être plus de soudaineté, économie de bazar et pratique commerçante traditionnelle sont confrontés à l'ouverture économique. L'approvisionnement se mondialise tandis que de nouveaux espaces de vente rencontrent les faveurs d'une clientèle toujours plus nombreuse et plus solvable.

Enfin, ces nouveaux lieux du commerce sont révélateurs des changements à l'œuvre dans la société algérienne. Loin des clichés sur le « choc des civilisations », la démographie et la structure familiale algérienne s'alignent sur le modèle nucléaire. Des femmes actives et salariées y négocient de nouveaux rapports avec leurs époux ou leurs compagnons, au moyen notamment d'un renouvellement des pratiques de la consommation domestique. 


\section{Les nouveaux lieux du commerce dans la ville algérienne : une modernité en construction}

\section{GUERRE INTÉRIEURE ET ACTIVITÉ COMMERÇANTE (DÉCENNIES I980-I990)}

Dans les villes algériennes, les espaces commerciaux sont historiquement localisés dans les rues. Outre des fonctions usuelles de circulation et de résidence, la rue est le lieu de l'économie de bazar. Considérée comme une ressource par les agents économiques, la rue est un espace valorisé dans la perspective d'un bénéfice marchand. Elle constitue en outre un espace de sociabilité particulièrement apprécié.

À partir des années 1990, les rues commerçantes algériennes perdent une grande part de leur statut. Sous la pression des courants islamistes radicaux, elles deviennent un espace de normativité renforcée, notamment pour les femmes. Dans un contexte de violence, la rue devient en outre un espace menaçant (attentats aveugles, massacres de civils, interventions des forces armées, etc.). Pour en fuir les risques, les populations s'enferment à leur domicile et remplacent l'interaction concrète et publique par de l'échange virtuel et domestique : les paraboles des chaines satellites puis les télécommunications informatiques remplacent pour un temps les douceurs et les petits chagrins de la vie urbaine. Durant les années de terreur, les citadins se forcent à l'introversion tandis que les espaces se fragmentent. Dix ans plus tard, les paysages urbains algériens portent encore la marque de cette période traumatique : doubles portes à l'entrée des logements, obstructions d'impasses, clôtures renforcées par des barbelés ou des parpaings, etc. De ce fait, l'animation commerciale urbaine perd de son importance, notamment dans les villes les plus confrontées à ces événements comme Alger, Constantine, Bouira ou Boummerdès. À rebours de tout un héritage d'urbanité méditerranéenne, les espaces publics y deviennent répulsifs, surtout dès la tombée du soir, ce qui nuit évidemment à l'activité commerciale.

Cependant, la « décennie noire » n’inverse pas totalement le cours des choses. Dès les années 1980, les autorités accordent des facilités en matière de visas, qui se poursuivent au cours de la décennie suivante. De jeunes Trabendistes en profitent pour importer les biens dont la société algérienne est alors privée. Ces jeunes gens parcourent l'Europe (principalement la France et l'Italie), à la recherche d'articles pour la personne (habillement, maroquinerie, bijoux de fantaisie, etc.) rapportés par des moyens de fortune et en petite quantité. Une fois entrés en Algérie, ces articles sont vendus sur des étalages improvisés, les Trabendistes ne payant pas d'impôts et ne déclarant pas leur activité. Malgré la violence de la guerre civile, la rue reste donc lieu de l'échange économique et de l'appropriation sociale. Mais ces interactions sont fugaces et elles opèrent dans des lieux à la fois fragmentés et éphémères.

\section{RETOUR À LA PAIX ET INSERTION DANS UNE ÉCONOMIE MONDIALISÉE :} VERS UNE « DubaïsATION » DU COMMERCE URbain (ANNÉES I990-2000)

Anticipant sur le retour de la normalité politique, l'économie algérienne s'ouvre sur les échanges mondiaux à partir des années 1990. En 1991, la libéralisation du commerce d'importation et son ouverture au secteur privé conduisent à la création de sociétés d'import-export. Avec la convertibilité du dinar, ces sociétés pratiquent les échanges sur de vastes échelles et sur de gros volumes: l'économie du conteneur se substitue rapidement à la valise clandestine des Trabendistes (Baugé, 2004; Mens, 2005). Les difficultés à obtenir des visas individuels pour la France accélèrent cette mutation à partir de la seconde moitié des années 2000 (Zouikri, 2009).

Cette nouvelle donne produit des espaces commerciaux d'un genre nouveau. Les souks traditionnels, situés au centre des villes denses, sont relayés par des pôles commerciaux souvent périphériques, où est pratiquée la vente en gros. Jusque là localisé dans la rue ou dans les souks, le commerce se déplace donc vers des lieux spécialisés selon le type de marchandise. C'est de cette période que datent les premiers Dubaï, qui voient le jour à Alger au début des années 
2000 à l'initiative de commerçants sétifiens. Il s'agit de lotissements regroupant plusieurs dizaines d'immeubles commerciaux dans lesquels sont proposées en grande quantité des marchandises étrangères importées à bas prix. Goûtant les métaphores, l'inventivité linguistique populaire a tôt fait d'imposer le nom générique de Dubaï à ces nouveaux lieux qui essaiment dans toute l'Algérie au cours des premières années de la décennie 2000. Nos enquêtes nous ont permis de rencontrer le commerçant qui, selon ses confrères, est le premier à avoir " acclimaté » cette dénomination à El-Eulma. Selon lui, le Dubaï « fait référence à la matérialisation des espaces commerciaux semblables à ceux de Dubaï, tant dans le regroupement des échoppes, la disposition des magasins, leur répétitivité, que par la masse des produits importés ainsi que la variété qu'ils présentent aux commerçants et clients».

Sur le plan morphologique, le Dubaï s’inspire du mall nord-américain adapté à la mode des villes du Golfe arabo-persique mais il fait également référence à la tradition des « grands magasins » à l'européenne. Sur le plan commercial, cette dénomination concerne l'origine des marchandises, dont une forte proportion transite par le port de Dubaï, où elle est négociée par les importateurs algériens. Enfin, sur un plan plus général, le terme Dubaï suggère un mixte de significations positives liées à la société de consommation : abondance, modernité, prix accessibles, ouverture sur la mondialisation, réactivité aux modes et aux «tendances ». La polysémie du terme semble donc traduire le dynamisme autant que l'indécision d'une société algérienne en voie d’insertion rapide dans l'économie mondiale de la consommation (photo 1).

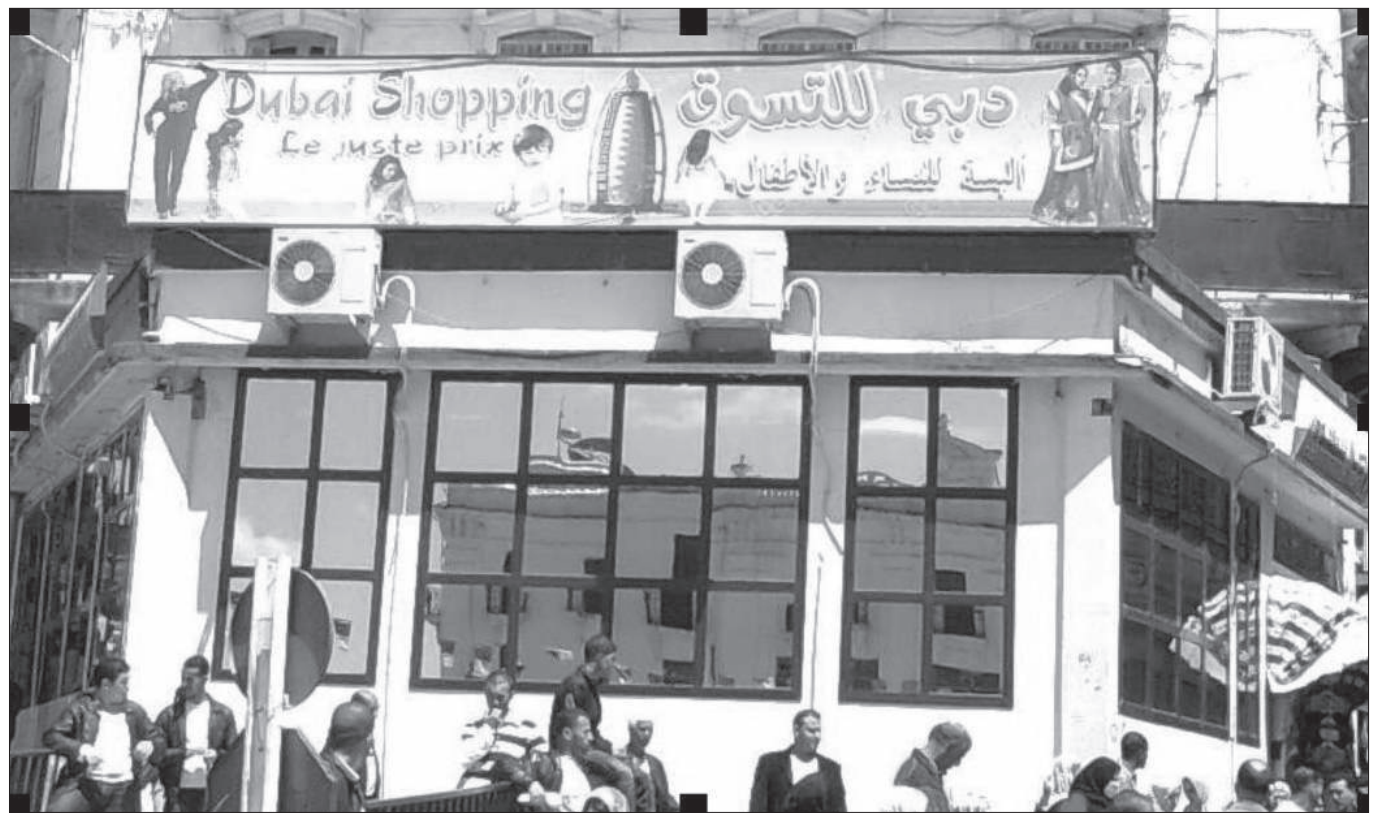

Photo 1 : La devanture d'un magasin au centre de Constantine : Dubaï Shopping. Le juste prix (P. Bergel, avril 2009) Cette photographie prise au centre-ville de Constantine (Place de la Brèche) montre la devanture d'un magasin de vêtements récemment installé. Les façades ornées de vitres réfléchissantes font référence à l'architecture commerciale des centres commerciaux du Golfe arabo-persique. Figurée sur l'enseigne, la silhouette de l'hôtel Burj Al Arab (situé à Dubaï) renforce la démonstration et justifie le nom du magasin. La superposition des langues (Arabe, Français, Anglais) et des allusions (« le juste prix » se réfère à une émission-jeu diffusée sur une chaine de télévision française) illustre à la fois l'ouverture et l'indécision d'une société algérienne en pleine mutation.

Store front-window in the city center of Castantina: "Dubai Shopping. Le juste prix" 
Les Dubaï sont alimentés par des circuits mondialisés. Produits localement jusqu'aux années 1990, les meubles arrivent désormais de Chine ou d'Italie tandis que l'électroménager et les équipements multimédias proviennent de Turquie, d'Europe centrale ou de l'ensemble du continent asiatique. L'internationalisation de l'offre fait accéder les consommateurs algériens à de nouvelles marchandises, les économies réalisées par le faible niveau des coûts de fabrication et la distribution en gros permettant d'abaisser les prix de vente de manière significative (photo 2). Côté demande, le pouvoir d'achat augmente, du fait de l'augmentation des prix du pétrole à l'exportation. La « dubaïsation » du commerce urbain en Algérie est donc liée à des changements macro-économiques de grande ampleur : mondialisation des circuits d'approvisionnement à l'importation, valeur de la rente pétrolière à l'exportation.

\section{NouveauX ESPACES COMMERCIAUX ET CHANGEMENTS SOCIO-CULTURELS}

Ces changements s'accompagnent de transformations toutes aussi profondes dans les pratiques de consommation. Les Dubaï remplacent la mobilité piétonnière par l'accès automobile, la chalandise traditionnelle par la consommation de masse. Sur le plan socioculturel, les pratiques de la déambulation commerciale se renouvellent et, plus largement, se structurent de nouveaux rapports à la marchandise (Barthes, 1957). À l'image de l'Europe occidentale des années 1960, se développe un nouvel idéal de vie centré sur « l'équipement de la maison » et le bonheur domestique de la famille nucléaire, révélant à son tour les mutations de la société algérienne contemporaine.

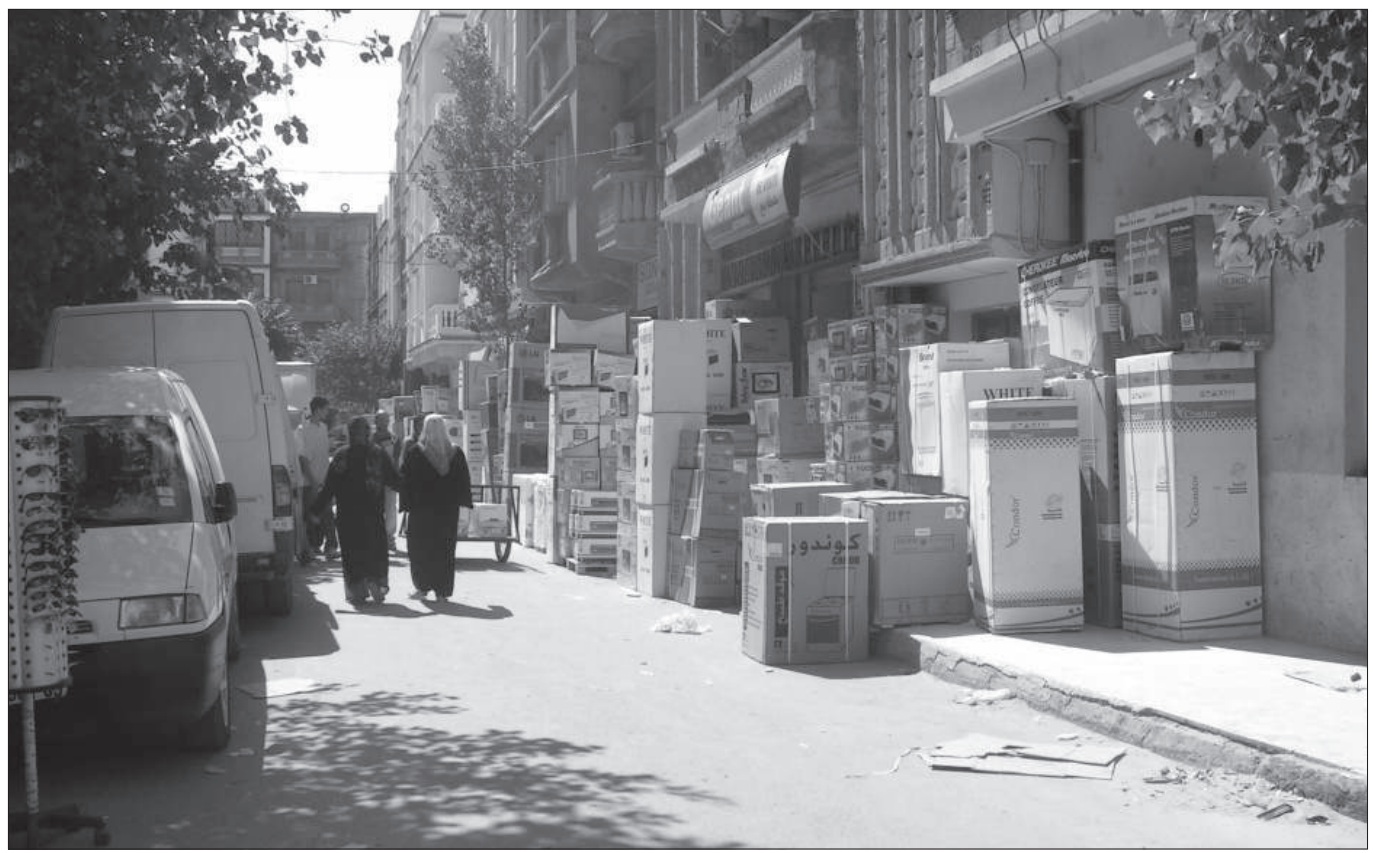

Photo 2 : Une rue encombrée de marchandises. Dubaï d'El-Eulma (P. Bergel, août 2008)

L'abondance des cartons d'emballage révèle les caractéristiques de ce nouveau commerce : approvisionnement en gros sur des marchés éloignés où les coûts de fabrication sont peu élevés. Cette organisation permet des prix de vente attrayants, doublés de marges confortables. Les commerçants bénéficient donc d'une affluence garantie, notamment féminine.

A street in El-Eulma cluttered with various wares 
Le commerce « dubaïsé » contribue donc à faire entrer les consommateurs algériens, de plus en plus solvables et motorisés, dans de nouvelles pratiques au contact d'une offre mondiale en cours de standardisation. Ces changements sont d'autant plus rapides en Algérie que, contrairement à d'autres pays du Maghreb ou du Proche-Orient, ils ont été retardés par plusieurs décennies d'isolement.

Dans ces nouveaux espaces commerçants, la société algérienne semble tourner la page de dix années de guerre civile. Des ménages éduqués et au pouvoir d'achat en hausse découvrent la déambulation consumériste ainsi que de nouveaux rapports à soi, aux autres et à la marchandise. Par ces pratiques, qui peuvent paraître ordinaires au consommateur occidental, se négocient de nouveaux rapports sociaux, notamment entre les hommes et les femmes. Ces nouveaux espaces commerciaux matérialisent donc une modernité en construction qui se développe dans un consensus relatif, indépendant des options philosophiques ou religieuses choisies par les ménages, les catégories sociales ou les générations (Haenni, 2005).

\section{Deux exemples de nouveaux espaces commerciaux dans les villes du nord-est algérien}

\section{MÉTHOde}

Les propos qui précèdent suggèrent que les mutations commerciales ne sont pas seulement une affaire économique. Produit par la mondialisation et la libéralisation des circuits d'importation, ce phénomène est également lié à des transformations qui l'apparentent à un «fait social total » (Mauss, 2007, [1924-1925]). Ne pouvant disposer de sources statistiques fiables, nous avons choisi la méthode de l'entretien semi-directif auprès des groupes de populations concernés par ces transformations. Au cours de l'année 2008, plusieurs campagnes d'enquêtes ont ainsi visé trois échantillons :

- Les clientèles ont été questionnées sur les motifs de leur fréquentation et sur les comparaisons qu'elles pouvaient opérer avec des lieux commerçants plus traditionnels. Une grande part des questionnements a été orientée sur l'usage des lieux : stationnements, déambulation, achats, etc.

- Les commerçants ont été interrogés sur leurs circuits d'approvisionnement, sur les biens et les services fournis à la clientèle (crédits informels à la consommation par exemple), sur les collaborations et concurrences avec leurs confrères, sur le montant et les modalités de leurs investissements. Nous leur avons posé des questions générales sur les modèles de consommation induits et des questions plus factuelles concernant l'aménagement des sites (stationnement, occupation de l'espace public) et leur protection (sécurité des personnes et des biens, transferts de fonds).

- Enfin, les employés de ces nouveaux commerces ont été questionnés sur leurs pratiques professionnelles, sur l'intérêt qu'ils y trouvent ainsi que sur les contacts qu'ils nouent avec la clientèle. Parmi ces employés, on trouve fréquemment de jeunes étudiants qui pratiquent cette activité de manière occasionnelle.

\section{Présentation des deux terrains d’enquête : Onama/El-Ghazi et El-Eulma}

Nos enquêtes ont porté sur deux espaces commerciaux baptisés Dubä̈ par les commerçants ou par leurs clients. Le premier est situé à une dizaine de kilomètres au sud-est de Constantine le long de la route nationale 3 vers El-Khroub (ONAMA/El-Ghazi). Le second est situé dans la ville d'El-Eulma, localisée à une trentaine de kilomètres à l'est de Sétif. Au premier abord, ces concentrations commerçantes peuvent être assimilées à des souks. Mais, contrairement à ces derniers, il s'agit de lotissements composés de rues se coupant à angle droit. Le long de ces rues, s'alignent des bâtiments commerciaux édifiés sur plusieurs niveaux. Ressemblant en miniature à des " grands magasins », ces derniers proposent une offre variée, notamment en matière d'équipements de la maison. 
À ONAMA/El-Ghazi, la route nationale 3 sépare deux emprises. Sur le côté sud, s'étend le lotissement d'ONAMA, édifié par un entrepreneur privé à proximité d'une entreprise d'État fabriquant du matériel agricole ${ }^{1}$. Édifié sans autorisation, le lotissement n'en est pas moins strictement organisé. Le propriétaire a confié une mission de maîtrise d'œuvre à un architecte-urbaniste qui a proposé un plan fixant la largeur des voies, les gabarits, les hauteurs, etc. L'objectif final est de constituer un Dubaï à l'image de celui d'El-Eulma. Sur le côté nord de la route, s'étend le lotissement plus récent d'El-Ghazi édifié quant à lui selon des procédures légales. Il est cependant impossible de le distinguer du premier, les formes et les ambiances y étant identiques. Selon des informations recueillies sur place, les prescriptions formulées pour El-Ghazi ont repris celles antérieurement expérimentées à ONAMA, ce qui explique les similitudes.

À terme, il est probable que la RN 3 se transformera en artère commerçante assurant la desserte d'un pôle commercial unique (photo 3). Il est d'ailleurs intéressant de remarquer que la toponymie anticipe sur cette tendance, puisque les clients comme les commerçants usent de la seule dénomination d'ONAMA pour désigner l'un ou l'autre des deux lotissements. Pour l'heure, la nationale demeure une voie de transit, ce qui rend délicat l'arrêt des véhicules ou les traversées piétonnes.

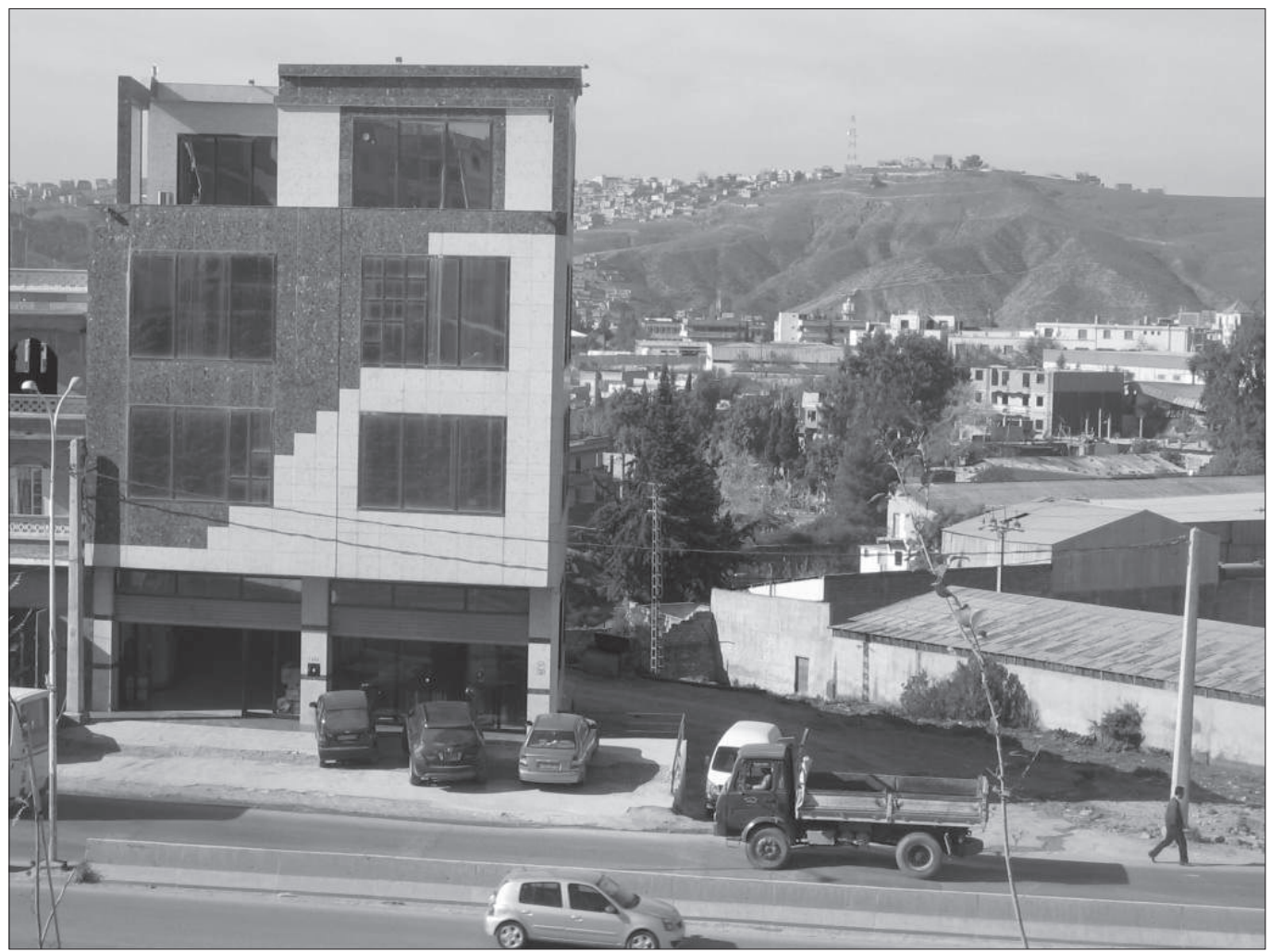

Photo 3 : La route nationale 3, vue en direction du sud-ouest. Dubaï d'ONAMA (N. Kerdoud, mai 2008)

Des immeubles commerciaux sont progressivement édifiés de part et d'autre de la RN 3, lieu d'un important trafic routier (au premier plan). Comme sur la photo 1, les façades font allusion aux centres commerciaux des pays du Golfe. Les aménagements permettant le stationnement ne sont pas encore exécutés, ce qui rend dangereuses les conditions d'accès.

Main road number three, south-western view, ONAMA Dubaï shopping mall 
Aucun aménagement n'étant encore prévu, des difficultés de stationnement apparaissent, notamment lors des périodes d'affluence. Pour prévenir les accidents, les commerçants d'ONAMA ont loué les services d'un « gardien du trottoir » dont la mission consiste à organiser la circulation et à assurer la sécurité des piétons.

Le Dubaï d'El-Eulma, quant à lui, est installé sur un lotissement de plusieurs dizaines d'hectares, situé à proximité de stations d'autobus et de taxis complétées par un immense parking. Les rues y sont de fait piétonnes, notamment aux périodes de grande affluence, même si elles ne sont pas explicitement interdites à la circulation (photos 2 et 4). Outre des magasins installés dans les rezde-chaussée ou les étages, elles accueillent des restaurants et des bars.

\section{L'ARCHITECTURE ET L'AGENCEMENT INTÉRIEUR AU SERVICE DE LA CONSOMMATION}

L'architecture propose des signes jusque là inemployées en Algérie. Contrairement à la disposition ordinaire du souk (rez-de-chaussée commercial surmonté d'étages d'habitation), les superficies commerciales occupent souvent l'ensemble des étages. Les façades sont travaillées en vue d'affirmer leur vocation, notamment au moyen de grandes superficies vitrées réfléchissantes (photos 1 , 3 et 4). Comme dans le souk traditionnel, le tronçon de rue devant la boutique fait office de lieu de stockage ou de surface de vente additionnelle (photo 2).

À l'intérieur, les espaces s'inspirent également du modèle du centre commercial du Golfe. Des escaliers garnis de dalles imitant le marbre sont censés garantir une atmosphère luxueuse (photo 5). Des systèmes de vidéosurveillance sont installés, des dispositifs anti-intrusion sont projetés. Contrairement aux souks, où les marchandises sont disposées sans mise en scène, ces magasins présentent des articles en situation, notamment pour le mobilier (photo 6). Des produits

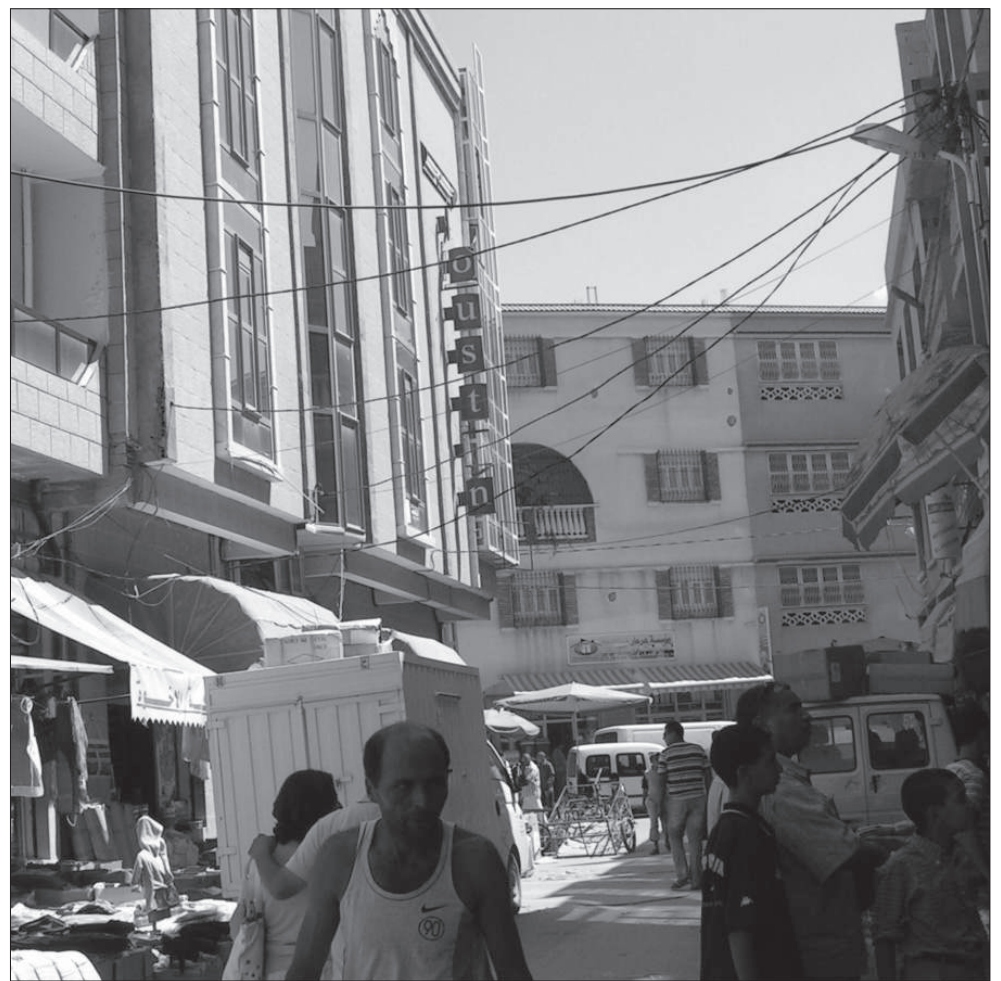

Photo 4 : Une rue commerçante à El-Eulma (P. Bergel, août 2008)

Les rues sont encombrées d'étals et de marchandises. À gauche, on distingue un magasin à étages à façade vitrée. Sur le bord droit de la photographie, on devine la terrasse d'un café-restaurant. in El-Eulma 
nouveaux sont en outre proposés, comme des cuisines intégrées importées d’Italie. Dans ce dernier cas, la similitude avec un magasin européen est totale.

Nos enquêtes ont révélé que ces partis-pris correspondent à des stratégies commerciales explicites. Le propriétaire du Pacha Center d'ONAMA a ainsi fait référence à ses voyages en Europe. Au cours de ses déplacements, il visite des centres commerciaux ou des grands magasins à l'intérieur desquels il prend des photos. Ces modèles l'inspirent pour recréer des ambiances similaires : " pourquoi pas un nouveau style à Constantine du genre Tati ou $C$ \& A? Les Constantinois ont bien droit à ce genre de commerces et d'édifices!»

Photo 5 : L'entrée du Pacha Center à ONAMA (N. Kerdoud, août 2008)

L'escalier monumental habillé de dalles imitant le marbre accrédite une impression de luxe qui valorise la pratique du « shopping ». Le prestige social lié à ce magasin constitue un motif de fréquentation, indépendamment des achats effectués.

Pacha Center entrance in ONAMA/EL-Ghazi

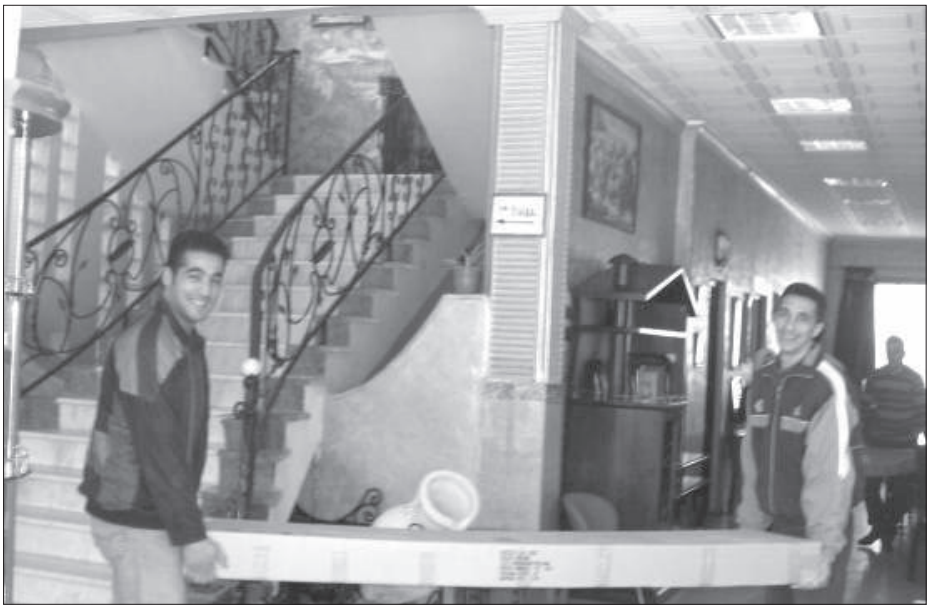

Photo 6 : Une exposition de meubles "en situation » dans le Pacha Center d'ONAMA/ElGhazi (P. Bergel, août 2008)

A situated furnitures exhibition inside Pacha Center. ONAMA/EL-Ghazi

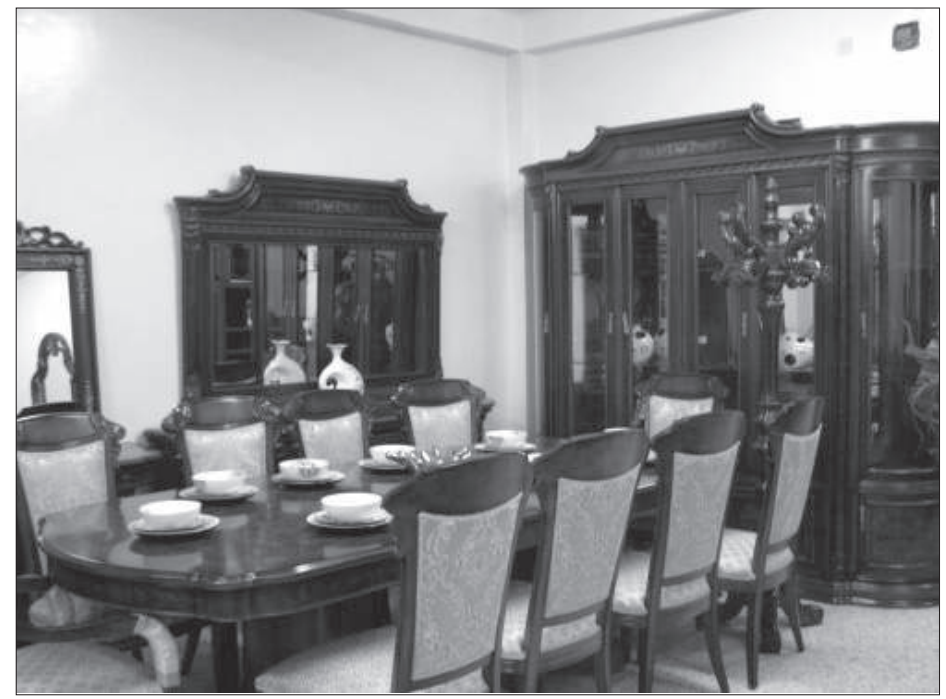


Pierre Bergel, Nadia Kerdoud

\section{Les nouveaux lieux du commerce, des « espaces discrets de la mondialisation»}

\section{NOUVEAUX LIEUX DU COMMERCE ET « MONDIALISATION PAR LE BAS »}

La mondialisation n'est pas exclusivement produite par des firmes multinationales hégémoniques. Alimentés par de jeunes entrepreneurs qui n’hésitent pas à traiter directement avec des fournisseurs asiatiques ou européens, les nouveaux lieux du commerce algérien sont façonnés par une «mondialisation par le bas » (Tarrius, 2002). Dans ces «espaces discrets de la mondialisation » (Pliez, 2008), s'affairent des « fourmis » plus que des grandes organisations. Les capitaux mobilisés ne sont pas levés sur les marchés internationaux mais générés par l'épargne individuelle, l'entraide familiale ou quelques coups de pouce de la bonne fortune. Les modes opératoires sont inventifs et ils se situent aux confins du légal et de l'illégal, s'apparentant à ceux qui sont utilisés dans le trafic des drogues ou des produits de contrefaçon.

Les marchandises proviennent d'Asie, d'Europe, du Moyen-Orient ou du Golfe arabo-persique. Elles transitent par la frontière tunisienne ou à partir des ports du nord (Skikda, Jijel, Annaba, Alger). Faible prix à l'achat, négociation sur de grosses quantités et circuits informels permettent d'abaisser les prix de détail, ce qui assure le succès de la formule. Ces circuits sont difficiles à pénétrer et les informations obtenues sont de ce fait fragmentaires. Compte tenu de l'avancement de nos enquêtes, nous ne pouvons pas prétendre à l'exhaustivité. Nous appuierons donc nos conclusions provisoires sur deux entretiens menés auprès de deux jeunes entrepreneurs d'ONAMA en avril et mai 2008. Mourad a choisi la voie légale pour importer des marchandises en provenance d’Europe alors que Hamid procède de manière plus informelle.

\section{DEUX IMPORTATEURS CONSTANTINOIS FACE À LA MONDIALISATION}

Mourad a hérité de la maison de ses parents qu'il décore et modernise avec de réels talents d'architecte d'intérieur. Une fois la transformation effectuée, il vend cette maison avec une confortable plus-value. Mourad raconte que l'aisance matérielle dont il bénéficie aujourd'hui date de cet épisode fondateur. "C'était le vrai coup financier de ma vie » confie-t-il. Il utilise ce capital pour développer une activité de promotion immobilière. Il achète des parcelles pour y faire construire des maisons luxueuses qu'il aménage et qu'il meuble. Sa clientèle est aisée et souvent issue des cercles politiques.

En 1990 il achète illégalement un terrain à ONAMA. Le bâtiment commercial qu'il y possède aujourd'hui (le Pacha Center) est achevé en 2001. Il emploie onze salariés, notamment des étudiants qui pratiquent cette activité de manière occasionnelle. Pour attirer la clientèle, Mourad propose des facilités de paiement en supportant un risque de crédit sur sa propre trésorerie. Il produit des meubles et des rideaux qu'il fait monter dans des ateliers locaux. "Une bonne partie de ce que vous avez vu, les rideaux et tout ce qui relève de la décoration, c'est nous qui les produisons " assure-t-il. Il importe également des produits asiatiques ou turcs mais c'est en Italie qu'il préfère passer ses commandes.

Utilisant la technique du transfert d'argent entre banques algériennes et italiennes, Mourad domicilie ses devises en Italie, ce qui rend plus commode le paiement sur place. «Moi, je n'ai pas besoin de faire appel à d'autres moyens pour mes importations » dit-il, faisant allusion aux pratiques illégales acceptées par beaucoup de ses confrères. Mourad justifie le choix de l'Italie par la proximité, qui raccourcit les délais de livraison (une semaine pour un conteneur entre les côtes italiennes et les ports de Skikda ou d'Alger). Une fois la marchandise commandée, Mourad passe à l'Agence italienne des transports maritimes où il s'assure du chargement des conteneurs. Revenu en Algérie, il utilise Internet pour vérifier le cheminement du navire. En quelques années, Mourad a développé une activité profitable par des voies légales. Si les affaires continuent de prospérer, il envisage d'ouvrir d'autres Pacha Center à Annaba, Oran ou Alger. 
Importateur de jouets et d'articles de sport, également installé à ONAMA, Hamid préfère quant à lui le circuit illégal. Une telle option nécessite un détour par la Tunisie d'où Hamid prend l'avion pour Shanghaï. Il fait lui-même le voyage afin de traiter directement avec son fournisseur chinois et le payer sur place, ce qui nécessite d'emporter de grosses sommes d'argent. Hamid raconte que la veille de son passage en Tunisie, il confie l'argent à un douanier algérien de ses connaissances. Celui-ci traverse la frontière sans être contrôlé et confie l'argent à un collègue tunisien. Hamid raconte: "Lorsque le jour se lève, je me présente aux douaniers, fais les formalités habituelles de passage des frontières et récupère une heure après, plus loin, mon argent du douanier qui l'a réceptionné la veille... Les Tunisiens se foutent absolument de la quantité d'argent que tu peux faire rentrer. L'important est que l'argent passe et dès que ceci est fait, le reste devient plus facile. " Pour assurer ses complicités, Hamid consent à des commissions occultes dont il n'a pas révélé le montant.

Une fois la frontière franchie, Hamid dépose l'argent dans une banque tunisienne, ce qui lui permet d'acheter un billet d'avion pour Shanghai. Dès son arrivée en Chine, il prend contact avec son fournisseur, auprès duquel il a préalablement passé commande grâce à Internet. Ne maîtrisant ni l'Anglais ni les langues chinoises, il fait appel à une Chinoise de confession musulmane pour la traduction. Parlant Arabe, Zineb accompagne Hamid durant son séjour : depuis le négoce jusqu’à la conteneurisation.

De retour à Constantine, Hamid est renseigné par un courrier DHL qui l'avertit dès que le porteconteneurs a quitté Shanghai. Il peut suivre le voyage sur Internet grâce au numéro du conteneur. Une fois débarquée dans un port algérien, la boîte est grutée et dédouanée par un agent transitaire agrée par l'État. À ce dernier, est confié un dossier mentionnant le registre de commerce, le certificat de conformité, le certificat de garantie du produit ainsi que les attestations nécessaires.

Le détour par la Tunisie permet donc à Hamid d'officialiser une activité d'importation effectuée à partir de capitaux passés en contrebande. Il permet en outre de payer moins de droits de douanes, moins d'impôts et moins de TVA à l'importation. Pour écouler ses produits, Hamid loue une boutique située à ONAMA. Malgré un loyer mensuel onéreux (70 000 Dinars algériens soient $700 €)$, il fait de gros bénéfices car les clients sont nombreux.

\section{LES FRONTIÈRES : UN CATALYSEUR DE MONDIALISATION}

Pour alimenter ces centres commerciaux avec des produits conformes à une demande en voie de globalisation, les entrepreneurs doivent inventer de nouveaux circuits commerciaux d'échelles mondiales. Dans une organisation administrative algérienne qui n'est pas encore adaptée à un tel changement, les entrepreneurs inventent des solutions afin de faire prospérer leurs affaires. Dans la tradition d'une activité marchande enracinée depuis longtemps dans la région, sont alors mobilisés des jeux complexes entre légal et illégal, formel et informel.

Sur les deux cas exposés, on remarque combien est crucial le rôle des frontières terrestres et maritimes. Cette discontinuité politique, par les asymétries qu'elle génère (Guillot, 2009), permet un double blanchiment : celui de l'argent au voyage aller, celui de la marchandise au voyage retour. Lorsque les opérations sont légales, la discontinuité frontalière permet d'accéder à des produits dont les critères de qualité et de prix sont attractifs pour une clientèle qui, sans cela, n'y aurait pas accès.

Produit de la mondialisation, le fonctionnement des nouveaux lieux de commerce est à son tour vecteur d'ouverture mondiale. Dans les boutiques, de nouveaux modes d'exposition de la marchandise impliquent la connexion des consommateurs avec l'échelle mondiale, notamment par le biais des magazines internationaux et des programmes télévisuels. Cette standardisation leur permet d'accéder à de nouvelles normes et à de nouveaux désirs de consommation. 


\section{Vers une occidentalisation des pratiques de consommation?}

\section{FAMILlE NUCLÉAIRE ET DÉVELOPPEMENT DE NOUVELLES PRATIQUES DE CONSOMMATION}

Beaucoup de jeunes ménages algériens souhaitent rompre la cohabitation avec leurs parents ou leurs collatéraux. À partir des années 1990, cette aspiration se concrétise avec le développement de diverses formules permettant l'accession à la propriété : logement social participatif (LSP), location-vente, acquisition à crédit, etc. Les ménages disposant de deux salaires peuvent alors massivement accéder à un logement autonome (Meghraoui-Chougiat, 2006). À l'image des foyers européens quelques décennies plus tôt, se développe alors un goût prononcé pour le mobilier, l'aménagement intérieur, la décoration. ONAMA/El-Ghazi et El-Eulma apparaissent comme des lieux commerçants qui répondent à cette demande nouvelle. Alors que les souks remplissent un rôle utilitaire, leurs boutiques proposent des marchandises qui font rêver. Jusqu'ici, ces décors de la vie domestique paraissaient inaccessibles, les foyers algériens pouvant seulement en deviner l'existence au travers des films et des fictions télévisées massivement diffusés sur les bouquets satellitaires $^{2}$. La multiplication des Dubaï concrétise ces images et transforme le rêve télévisuel en réalité. Le Pacha Center est ainsi perçu comme un magasin différent : "C'est propre, c'est clair et tous les produits sont beaux ici » remarque une femme interrogée à l'intérieur du magasin.

La fréquentation d'El-Eulma témoigne également de cet attrait. Un comptage aléatoire effectué sur 141 véhicules en stationnement sur le parking central (septembre 2008) montre la diversité géographique de la clientèle, 27 Wilayas étant représentées (tableau 1). Les immatriculations locales (Sétif, Jijel) ou proches (Annaba, Constantine, Guelma) regroupent environ les deux tiers du total mais on note également la présence de véhicules originaires de destinations plus lointaines : nord-ouest algérien (Mostaganem, Tlemcen, Tiaret), est (El Tarf, Oum-El-Bouaghi), région-capitale (Alger, Boummerdès), sud (Laghouat, Adrar). En outre, neuf véhicules sont immatriculés en Europe. La clientèle d’El-Eulma passe plusieurs heures à faire du « shopping » puis elle se restaure avant de quitter le site pour retourner vers ses lieux de résidence. Les photos 7 et 8 , respectivement prises vers midi puis aux environs de quinze heures montrent le volume et les rythmes de cette fréquentation. Pour accéder à un rêve consumériste qui leur semble précieux, certains clients d'El-Eulma n'hésitent donc pas à parcourir de longues distances, dans des conditions de voyage souvent inconfortables.

\begin{tabular}{|l|c|}
\hline \multicolumn{1}{|c|}{$\begin{array}{c}\text { Origine des véhicules } \\
\text { (par grandes régions et exemples de Wilayas) }\end{array}$} & Nombre \\
\hline Centre (Alger, Bordj-Bouarirdj, Boummerdès, M’sila) & 17 \\
\hline Nord-Est (Annaba, Batna, Constantine, Guelma, Sétif, Skikda, etc.) & 103 \\
\hline Nord-Ouest (Aïn-Temouchent, Chlef, Oran, Mostaganem, Tiaret) & 8 \\
\hline Sud (Adrar, Biskra, El-Oued, Laghouat) & 4 \\
\hline Immatriculations européennes & 9 \\
\hline TOTAL & $\mathbf{1 4 1}$ \\
\hline
\end{tabular}

Tableau 1 : Provenance des véhicules stationnés sur le parking central d’El-Eulma (septembre 2008, en semaine ; document établi à partir du relevé des plaques d'immatriculation des véhicules individuels)

Origins of vehicles parked in the El-Eulma central car park

2. Plus encore que leurs homologues français, les programmes de fiction diffusés par les nombreuses chaines égyptiennes et turques jouent un rôle déterminant dans la diffusion de ces normes. Ces programmes rivalisent pour présenter des logements luxueux associés aux toilettes féminines les plus raffinées. L'idéal domestique s'y conjugue avec une nouvelle place de la femme dans la société musulmane, même si l'argument de ces soap opera est souvent d'une médiocrité affligeante... 


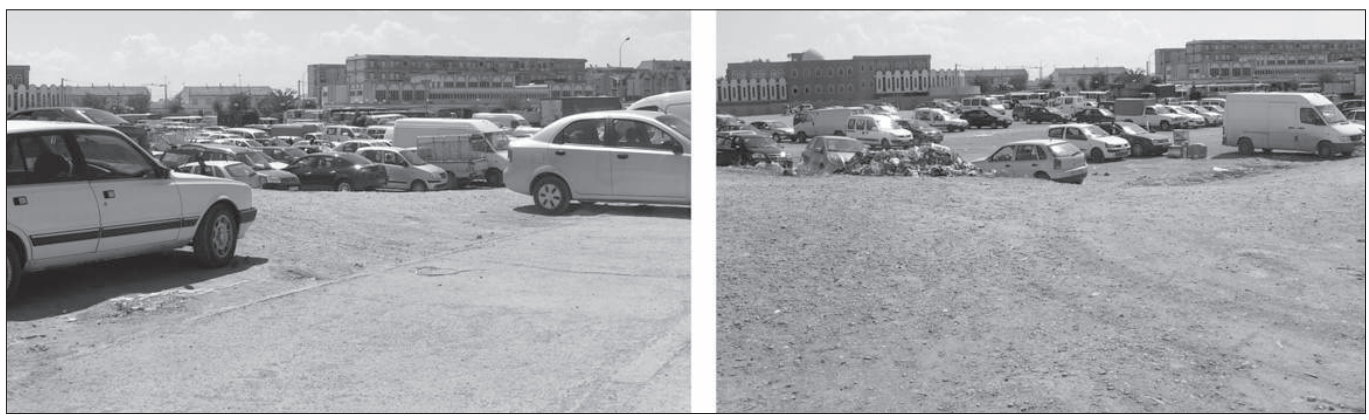

Photos 7 et 8 : Le parking central d'El-Eulma aux environs de midi puis vers 15 heures (Auteur : P. Bergel, août 2008)

Les deux photographies donnent une idée des rythmes de fréquentation du Dubaï d'El-Eulma. Partis en fin de nuit, les clients arrivent à El-Eulma en fin de matinée et déambulent dans les rues commerçantes jusqu'au début de l'après-midi. Les achats sont interrompus par une pause-déjeuner dans un des restaurants présents sur le site. Les retours s'effectuent entre 15 et 16 heures.

El-Eulma central car park around noon and then about three pm

\section{«Shopping » ET RÊVE Domestiques}

Quelle motivation pousse la clientèle à fréquenter ces nouveaux lieux du commerce? L'enquête réalisée à ONAMA/El-Ghazi au printemps et à l'été 2008 a permis de mener 20 entretiens (10 couples, 8 femmes venues seules ou avec leur fille, 2 jeunes filles), dont nous extrayons les extraits les plus significatifs. Les adultes interrogés ont un âge compris entre 20 et 61 ans.

Les raisons de la fréquentation tiennent au type de marchandise (équipement de la maison) et au projet de construire un espace familial autonome. Pour Fairouz (professeur d'Anglais) et Ali (infirmier à l'hôpital de Constantine), tous deux âgés de 35 ans et parents de trois enfants, "venir à ONAMA et El-Ghazi c'est penser dès à présent à la concrétisation d'un rêve : celui d'avoir un beau logement à nous. » Fairouz et Ali confient qu'ils viennent souvent à ONAMA en compagnie de leurs enfants. Ils stationnent leur automobile le long de la RN3, Fairouz tient ses deux filles par la main tandis qu'Ali surveille le garçon. Fairouz raconte que leur intérêt pour ONAMA est lié à des projets d'équipement pour leur futur logement. Le couple a habité pendant dix ans avec les parents d'Ali dans un appartement situé au centre-ville de Constantine. Ils ont pu quitter ce premier domicile grâce à l'aide de la mère de Fairouz, qui a prêté un logement. Le couple deviendra prochainement propriétaire au moyen d'une formule de location-vente. Au cours de la visite, c'est l'épouse qui prend l'initiative. Elle commence par contempler les objets exposés puis elle invite son mari à donner son avis. Ils discutent ensuite afin de fixer le montant de leurs dépenses du jour. «Passer d'une échoppe à une autre, admirer les nouveautés nous permettra non seulement de faire quelques affaires pour bien équiper notre maison, mais aussi de passer quelques temps et se changer les idées » confie Fairouz.

Amies et voisines, Rofia (23 ans) et Samia (25 ans) se rendent deux fois par semaine à ONAMA/ El-Ghazi. Elles apprécient les articles exposés mais trouvent les prix trop élevés. Rofia compte se marier mais comme elle ne travaille pas, elle a beaucoup de mal à préparer son trousseau. Elle fait du shopping à ONAMA afin de comparer les prix et voir si elle peut faire quelques bonnes affaires. Dans ce deuxième cas, la fréquentation d'ONAMA est liée à la déambulation et au "shopping " plus qu'à l'achat. On peut néanmoins faire l'hypothèse qu'un tel jeu avec la marchandise n'est pas innocent. En en multipliant les occurrences, ces deux jeunes filles se construisent une représentation de leur vie familiale future. La contemplation des objets exposés, le contact (même fugace et muet) avec de jeunes couples engagés dans une démarche du même genre, doivent puissamment contribuer à l'acculturation vis-à-vis du modèle familial traditionnel comme à la transformation des goûts et des pratiques de consommation. 


\section{Conclusion}

L'enquête de terrain révèle des correspondances entre trois mouvements. Le renouvellement de l'architecture et de l'urbanisme commercial s'accompagne de nouvelles sources d'approvisionnement. Les nouveaux espaces commerciaux s'insèrent dans des réseaux mondialisés d'accès à la marchandise, que ce soit de manière légale ou illégale. Ces dynamiques sont liées à des changements dans les pratiques de consommation, qui concernent les ménages et plus encore les femmes. Dans les Dubaï, ces dernières expérimentent des pratiques de shopping à l'occidentale et affirment leur intérêt pour le mobilier et les articles de la maison. Associant les époux et les fiancés, elles déplacent progressivement les frontières entre mondes masculins et sphères domestiques contrôlées par les femmes.

Ces trois mouvements semblent à leur tour liés à une mutation des structures sociales et familiales, ce que nos enquêtes laissent entrevoir de manière indirecte. Pour cause de crise du logement, les jeunes ménages ont maintenu une cohabitation avec leurs aînés ou leurs collatéraux jusque dans les années 1990. Souvent subie, cette cohabitation est désormais rejetée au profit de la famille nucléaire, tandis que l'action des pouvoirs publics en faveur de l'accession à la propriété des jeunes ménages bi-actifs donne des moyens matériels à cette nouvelle autonomie. Dans des logements de petite taille mais de plus en plus confortables, les « nouvelles familles » algériennes regroupent les seuls parents et un nombre restreint d'enfants, du fait de la généralisation des pratiques contraceptives ${ }^{3}$.

Partielle, cette enquête ne permet pas d'affirmer avec certitude que les nouveaux espaces commerciaux concourent à l'alignement des ménages algériens sur des standards occidentaux diffusés par l'ouverture mondiale des économies, des sociétés et des contenus télévisuels. L'usage du véhicule individuel, le shopping familial, la convergence entre consommation et loisirs rapprochent toutefois leurs comportements de ceux enregistrés depuis trois ou quatre décennies dans les pays du Nord. Contrairement à des travaux qui ont pu mettre l'accent sur l'accroissement des différences entre pays développés et sociétés arabo-musulmanes (Huntington, 2000), l'étude de terrain présentée dans cet article permet de soutenir l'hypothèse inverse. Les nouveaux lieux du commerce algérien apparaissent comme des lieux de convergence entre deux mondes. Par les changements qu'ils génèrent et qu'ils révèlent, ils contribuent à l'ancrage de la société algérienne dans les courants de la mondialisation économique et de l'uniformisation socio-familiale. Il faut toutefois nuancer cette affirmation en précisant que ces dynamiques ne concernent vraisemblablement qu'une part encore minoritaire de la population algérienne.

\section{Bibliographie}

BAugé F., 2004. Le pouvoir algérien cherche à endiguer la contrebande. Le Monde, $1^{\mathrm{er}}$ juin 2004.

Barthes R., 1957. Mythologies, Paris, Seuil, 247 p.

Côte M., 2005. L'Algérie, Constantine, éd. Média-Plus, 253 p.

Guillot F., 2009. Les asymétries frontalières. Essai de géographie sociale et politique sur les pratiques sociales et les rapports sociaux. Les cas États-Unis/Mexique, Espagne/Maroc, Israël/Liban/Palestine, Thèse de géographie, soutenue le 25 novembre 2009 à l'Université de Caen Basse-Normandie, 497 p.

Hancock C., 2008. Discutante de la conférence de G. Di Méo : «L'individu, le corps et la rue globale. Quelles constructions des citadin(e)s, des urbain(e)s? ", Colloque Où en est la rue face à la globalisation? Standardisation, singularisation, régulation. UMR ADES, Bordeaux, Maison des Suds, 27 et 28 novembre 2008.

3. Dans les enquêtes en face-à-face, les femmes individuellement interrogées par la seule collègue algérienne ont volontiers parlé de ce volet de leur vie intime. Sur l'échantillon qui est le nôtre, il ressort que ces femmes ont massivement recours aux techniques contraceptives, indépendamment des options religieuses et des choix vestimentaires arrêtés par le couple (tenue occidentale ou voile). 
Haenni P., 2005. L'islam de marché, Paris, Seuil, coll. « La République des idées », 108 p. Huntington S., 2000. Le choc des civilisations, Paris, O. Jacob, 545 p.

Mauss M., 2007. Essai sur le don: forme et raison de l'échange dans les sociétés archä̈ques, Texte extrait de L’Année sociologique, seconde série, 1924-1925, tome I. Paris, Presses universitaires de France, 248 p.

Meghraoui-Chougiat N., 2006. Quel habitat pour l'Algérie? La nouvelle ville de Constantine, Constantine, éd. Saïd Hannachi Média-Plus, 207 p.

Mens Y., 2005. "Profession trabendiste ». Alternatives internationales n² 20, janvier 2005.

Pliez O., 2008, « Des jeans chinois dans les rues du Caire ou les espaces discrets de la mondialisation », Mappemonde 88 (2007/4), p. 1-14.

Troin J.-F. (dir.), 1985. Le Maghreb, hommes et espaces. Paris, A. Colin, coll. « U », 360 p.

TARrius A., 2002. La mondialisation par le bas. Les nouveaux nomades de l'économie souterraine, Paris, Balland, $168 \mathrm{p}$.

Zouikri A., 2009. El Milia. Profession : trabendiste, L'écho de Jijel, $1^{\text {er }}$ mars 2009.

Cet article a été reçu le 7 avril 2010 et définitivement accepté le 13 décembre 2010. 